\title{
Electronic Phase Shifting in Multipulse Rectifier
}

\author{
Yevgen I. Sokol (Professor, National Technical University of Ukraine "Kharkiv Polytechnic Institute"), \\ Volodymyr V. Zamaruiev (Professor, National Technical University of Ukraine "Kharkiv Polytechnic Institute"), \\ Volodymyr V. Ivakhno (Professor, National Technical University of Ukraine "Kharkiv Polytechnic Institute"), \\ Yurii S. Voitovych" (Graduate student, National Technical University of Ukraine "Kharkiv Polytechnic Institute")
}

\begin{abstract}
This paper presents a novel converter which can reduce the harmonics like the conventional multipulse converters with input three phase transformer. To reduce total harmonic distortion of input current and improve the weight and size of converters, it is suggested to use multi-pulse rectifiers with an electronic phase shift. The basic module is a 6-pulse rectifier on fully controlled switches with the reverse blocking ability. Switching frequency either coincides or is twice the power frequency. The proposed solutions allow refusing from the electromagnetic phase-shifting devices (power transformers or auto-transformers) and thereby significantly reduce the weight of the device. Unlike power factor correction systems with highfrequency modulation, the proposed converters are significantly different, as they have better electromagnetic compatibility and the virtual absence of dynamic switching losses of power switches.
\end{abstract}

Keywords - Electronic phase shifting; Multipulse rectifier; Power quality; Power system harmonics; THD; Total harmonic distortion; Unit power coefficient .

\section{INTRODUCTION}

The vast amount of consumed electrical energy is used in a modified form. This is due to the requirements of consumers to change the characteristics of the voltage supply: frequency, shape or volume. The most frequently used converters are from $\mathrm{AC}$ to $\mathrm{DC}$ or $\mathrm{AC}$ to $\mathrm{AC}$ with $\mathrm{DC}$ link. In both cases, the converter includes AC/DC convertor, in the simplest case - a rectifier. Converters connected to $\mathrm{AC}$ power are characterized by several parameters: power consumption, power factor and harmonic content of consumed current from the sine wave. The first two parameters determine the loss in the supply line, and the third - the impact on consumers of the converter, which are also connected to this line [1].

6-pulse and multipulse uncontrolled and controlled rectifiers, three-phase active rectifiers [2], various options of VIENNA Rectifiers [3], etc. are widely used. The main disadvantage of classical uncontrolled rectifiers is significant coefficient of harmonic distortions of the current consumed from the network. In the case of a controlled rectifier, a multiple factor of power is added. Multi-pulse rectifiers $(p>6)$ reduce total harmonic distortion (THD) of input current, but require the use of phase-shifting transformers with a nominal installed capacity, which significantly degrades mass results of rectifiers' performance. Reduction of the rectifiers' weight can be achieved using autotransformer phase shifters [4]. Solutions of transformer phase shifters having less weight compared to the above [5] are known. In all the above cases, the weight of the transformer equipment is essential. Dynamic losses of switching are negligible small.

Radical weight reduction is achieved by the magnetic transducer elements in semiconductor PFC [2], [3]. For all PFCs, which use technology that increases the PWM, unity power factor and low THD input current at low frequencies areas (up to the 50th harmonic of mains frequency) by shifting the generated harmonic current in the high-frequency area is indicative. Modern power semiconductor devices can increase the switching frequency above the 50th harmonic of the mains frequency and up to several tens of kilohertz (depending on the power and voltage). These converters have excessive power loss due to switching losses in the power switches. In connection with the increased switching frequency, provision of electromagnetic compatibility of electronic devices (EMC) becomes relevant.

Direct normalization of generated harmonics in the mains current is produced at frequencies $100 \mathrm{~Hz}$ to $2500 \mathrm{~Hz}$ (at a frequency of $50 \mathrm{~Hz}$ mains) [1], [6]-[8]. On the other hand, it is necessary to ensure EMC, that is why current standards require the control of interferences in the frequency range of $2 \mathrm{kHz}$ to $150 \mathrm{kHz}$ [9], [10]. Compliance with EMC involves indirect rationing of generated current harmonics in the mains.

The need to ensure EMC, leads to the use of input filters in AC/DC converters, designed to operate at frequencies of a few to hundreds of kilohertz, the application of structural measures to reduce the level of symmetrical interference in a power supply. Such measures worsen the technical and economic characteristics of high power factor correction.

The article describes the use of multipulse rectifier, which does not require phase shifting transformer and is used to create a phase shift control means. The switching frequency is commensurate with the frequency of the network.

\section{The Operating PRINCIPLE OF THE PROPOSED AC/DC CONVERTER}

\section{A. Electronic Phase Shifting}

Multipulse systems are used to reduce the harmonic distortion coefficient of rectifier input current. The use of phase-shifting transformer and multiple 6-pulse uncontrolled rectifiers give the possibility to eliminate or reduce the selected current harmonics due to the vector addition [11]. To minimize current THD, the phase shift angle $\psi$ between the rectifier input

\footnotetext{
* Corresponding author.

E-mail: voitovychys@gmail.com
} 
voltages is selected in accordance with (1) and depending on the number $n$ of 6-pulse rectifier.

$$
\psi=\frac{60}{n} \text {. }
$$

Since in uncontrolled rectifier the displacement factor is equal to unity, and the phase shift of the voltages for a multipulse rectifier is provided by the transformer, the displacement factor of multipulse rectifier is also equal to unity.

In [12] it was proposed to assure a phase shift corresponding to a 12-pulse rectifier by using two 6-pulse controlled rectifiers connected to the same power source without using a transformer. To compensate the positive phase shift inherent in the conventional controlled rectifier (SCR), the second rectifier operates with negative control angles that have been ensured by commutation unit. Such a scheme is equivalent to a 12-pulse rectifier. Vector diagram of the input rectifier currents is shown in Fig. 1.

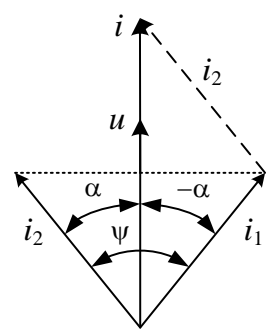

Fig. 1. Vector diagram of the input currents of equivalent 12-pulse rectifier with electronic phase shifting.

The angles of the control are $\alpha=\psi / 2$. The input current $I$ of the converter matches in phase with the input voltage of the rectifiers.

$$
\vec{I}=\vec{I}_{1}+\vec{I}_{2}
$$

The simplest circuit diagram of 12-pulse rectifier with electronic phase shifting (REPS) is shown in Fig. 2.

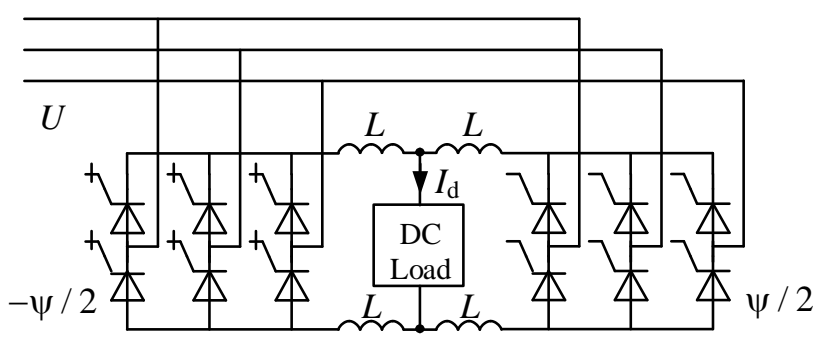

Fig. 2. Simplest circuit diagram of 12-pulse rectifier with electronic phase shifting.

Voltage waveforms in GTO and SCR rectifiers, which are part of 12-pulse rectifier, are presented in Fig. 3. For getting negative angles of control, fully controlled switches [11] IGBT, GTO, etc. with reverse blocking capability should be used. a)

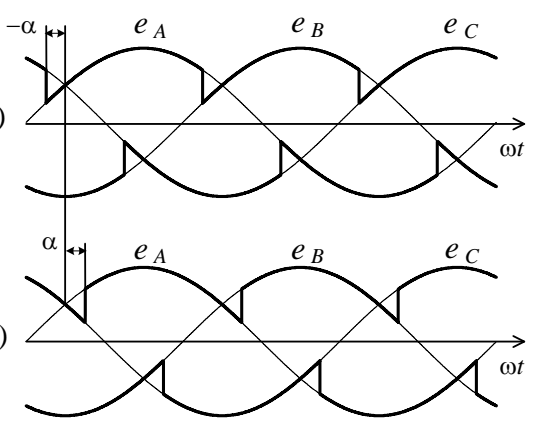

Fig. 3. Voltage waveforms in 12-pulse rectifiers circuit: a) GTO rectifier, b) SCR rectifier.

\section{B. Multipulse Rectifier With Electronic Phase Shifting}

Using the considered principle of the multipulse rectifier construction with a phase shift, which is formed by means of control, we can represent a generalized functional diagram of the rectifier (Fig. 4). The diagram contains $n=2 k$ rectifier units and equivalent $6 n$-pulse rectifier. The angles of control are computed in accordance with (1). The number $n$ is not necessarily even $(n=2 k)$. In the case of $n=2 k+1$ one of the rectifiers is operating with zero-angle control, and in some cases uncontrolled (diode rectifier) rectifier can be used.

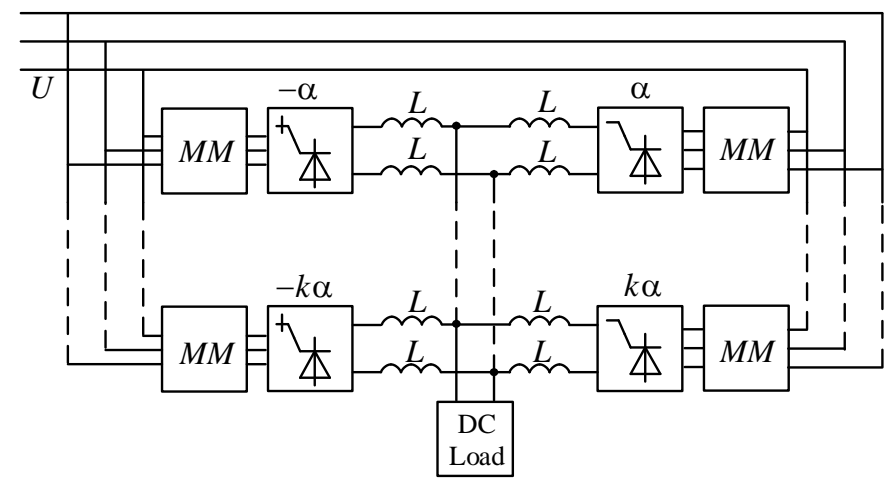

Fig. 4. Generalized functional diagram of the rectifier with electronic phase shifting. MM - matching module.

Vector diagrams for multi-pulse REPS with even and odd $n$ are shown in Fig. 5. In the case of even $n$,

$$
\left|\alpha_{1}\right|=\left|\alpha_{2}\right|=\psi / 2,\left|\alpha_{2 k}\right|=\left|\alpha_{2 k-1}\right|=k \psi+\psi / 2 .
$$

In the case of odd $n$,

$$
\left|\alpha_{1}\right|=\left|\alpha_{2}\right|=\psi,\left|\alpha_{2 k}\right|=\left|\alpha_{2 k-1}\right|=k \psi, \alpha_{2 k+1}=0 .
$$

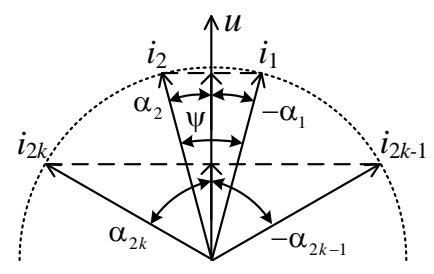

a) b)

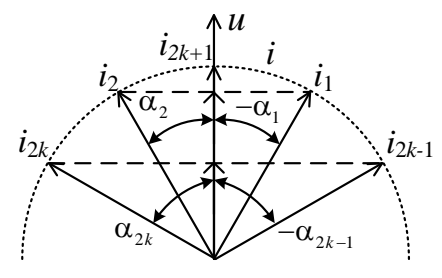

Fig. 5. Vector diagram of the input currents at electronic phase shifting: a) $n=2 k$, b) $n=2 k+1$. 
As can be seen from Fig. 5, at $n>2$ the currents of rectifiers with different angles of control are different. To equalize the output currents of the rectifiers, increasing input voltage of rectifiers that operate at high angles of control as well as decreasing input or output voltage of rectifiers, working with smaller angles of control, can be used. On the generalized functional diagram of a rectifier with an electronic phase shift, this function is performed by matching modules (Fig. 4). Increasing or decreasing of voltage can be obtained using lowpower matching autotransformers. It can be shown that the installed capacity of the autotransformer for the 18-pulse rectifier is $2 \%$ to $4 \%$ of the power load.

If all switches in rectifier modules in Fig. 4 are made to be fully controllable, then the rectifier module can be made unified, thus it is possible to remove the matching modules (matching autotransformers). In this case, to reduce the output voltage of the unified rectifier module without changing the current shift angle according to (1), a low frequency pulsewidth modulation may be applied. It is appropriate to use IGBT (Fig. 6) as switches for modules at hundreds of kilowatt power for the high voltage modules - reverse blocking GTO.

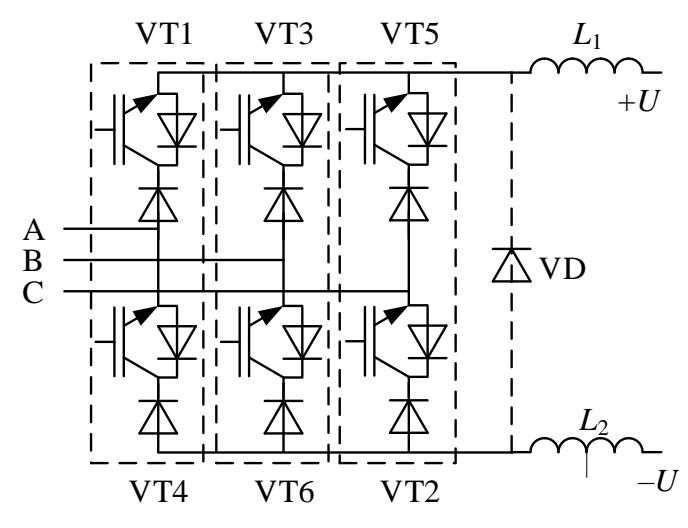

Fig. 6. Unified rectifier module.

Consider a switching algorithm for the semiconductor switching devices (Fig. 7) at the interval between the natural commutation points $c p_{1}-c p_{3}$. Unified rectifier module operates with control angle $\alpha$. The transistor VT1 is switched at point $p_{2}$, wherein $c p_{1}-p_{2}=\Delta \alpha_{2}$. The final switching off of this transistor is made at point $p_{5}$, and $c p_{3}-p_{5}=\Delta \alpha_{1}+\Delta \alpha_{2}$. Unlike a simple algorithm (Fig. 3a), switching off a power transistor will be performed earlier (on $\Delta \alpha_{1}$ ) and switching on later (on $\Delta \alpha_{2}$ ). Generally $\Delta \alpha_{1} \neq \Delta \alpha_{2}$. In interval $p_{3}-p_{4}$, switches of anode group are switching off. In the presence of the diode VD, which conducts the current, in its absence - the transistor VT4 must be switched, and that provides a flowing of current inductance. Similarly, switches of rectifier modules that work with the lagging control angle are commutated. Diode VD in Fig. 6 is optional. Its functions of creating a loop for inductance current may be realized while switching on the pair transistors from anode and cathode groups of the rectifier simultaneously. It can be seen that the minimum number of commutations (turn on or off) for the transistor of unified module is equal to two in the presence of the diode VD or four in its absence at the one period of power supply voltage.

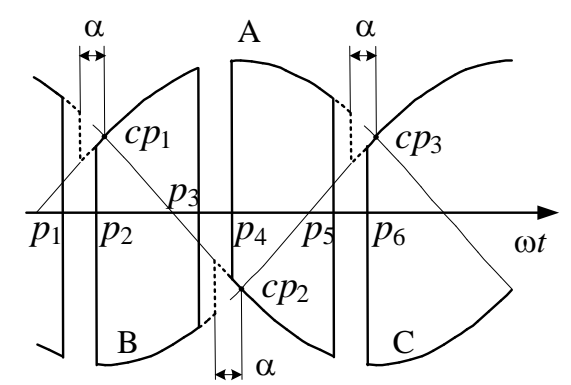

Fig. 7. Timing diagrams of unified rectifier module.

Since the switching frequency of the unified rectifier module is comparable with the frequency of the network, the dynamic losses can be neglected.

\section{TOTAL HARMONIC CURRENT DISTORTION}

Harmonic content analysis of the multipulse REPS input current was carried out by using Fourier series expansion of the current curves obtained under the assumption of the equal rectifier modules output current, perfect filtration and the unit of the first current harmonic. The calculation results for the current THD from a pulse number rectifier are shown in Fig. 8.

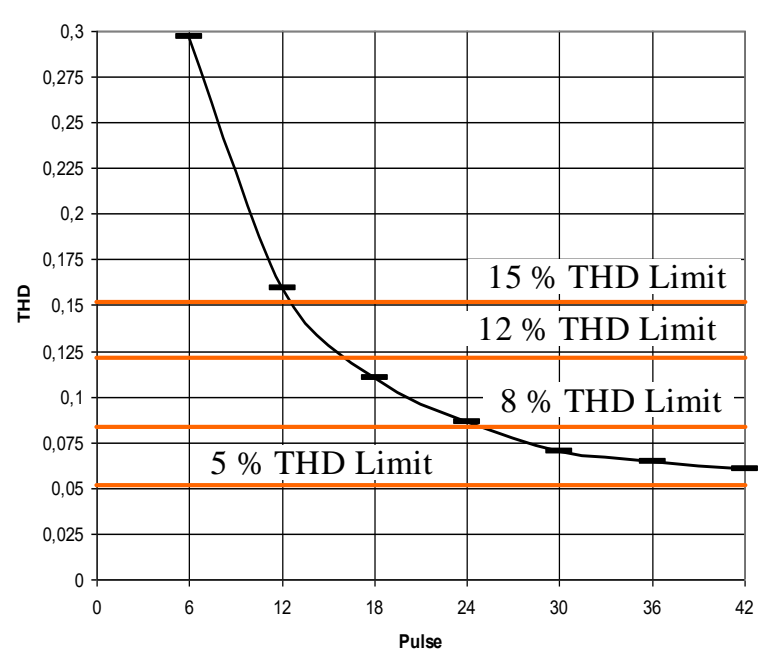

Fig. 8. Dependence of current THD on rectifier pulse.

Considering the data from Fig. 8, it can be concluded that the rectifiers with pulse-number more than 30 comply with the requirements [1], except for the weakest power lines $\left(I_{\mathrm{SC}} / I_{L}<20\right.$, the ratio of short-circuit of the power line to the load current). When rectifier pulse-number is 24 , it requires the input filter with an installed capacity of less than $1 \%$ of the power load. When the characteristics of the power line is $50<I_{\mathrm{SC}} / I_{L}<100$, rectifiers with pulse-number 18 and more comply with the requirements [1]. Almost the same results were obtained in the simulation in Simulink environment.

The minimum value of THD of current is achieved with a phase shift determined by (1). Considering the current vector diagram of 12-pulse rectifiers (Fig. 1), it can be concluded that to satisfy condition (2) with a unit displacement factor, only a single value of the control angles is possible $(\alpha= \pm \psi / 2)$. The use of a 24-pulse rectifier corresponds to the vector diagram of the currents shown in Fig. 5, a). In this REPS two pairs of 
rectifier bridges with symmetric control are used. Consequently, a unit displacement factor can be realized with the pair wise fulfillment of condition (2), in the simplest case, under the conditions

$$
\left|\alpha_{1}\right|=\left|\alpha_{2}\right|, \quad\left|\alpha_{3}\right|=\left|\alpha_{4}\right| .
$$

The values of the control angles $\alpha_{i}$ depend on the shift angles $\psi_{i}$ between the voltages of the rectifiers, at that the values of $\psi_{i}$ may differ from the recommended $\psi(1)$

$$
\left|\alpha_{1}\right|=\left|\alpha_{2}\right|=\psi_{1} / 2, \quad\left|\alpha_{3}\right|=\left|\alpha_{4}\right|=\psi_{1} / 2+\psi_{2},
$$

hence, the angles (6) may not correspond to (3).

THD value of the input current of the 24-pulse rectifier has a pronounced extremum depending on the values of the shift angles $\psi_{i}$ (Fig. 9, a). When minimum THD of the input current is forming, the shift angles are interrelated and can be matched to change up to 0.1 Radians (Fig. 9, b)).

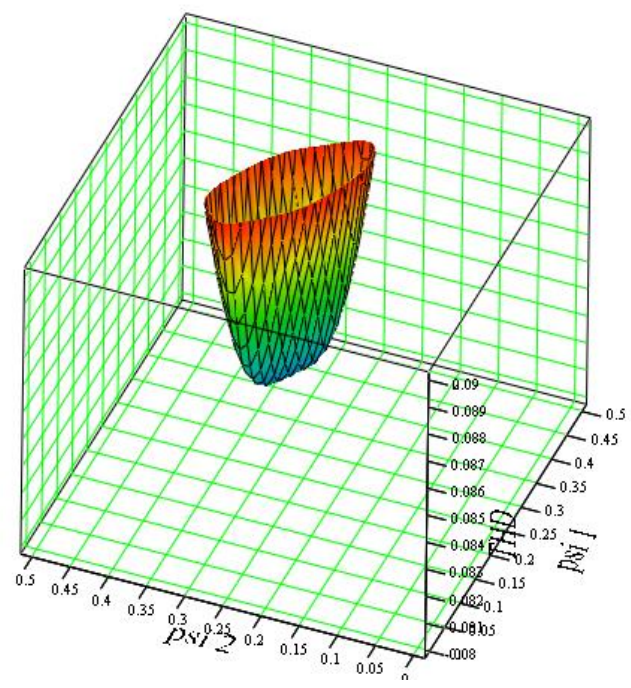

a)

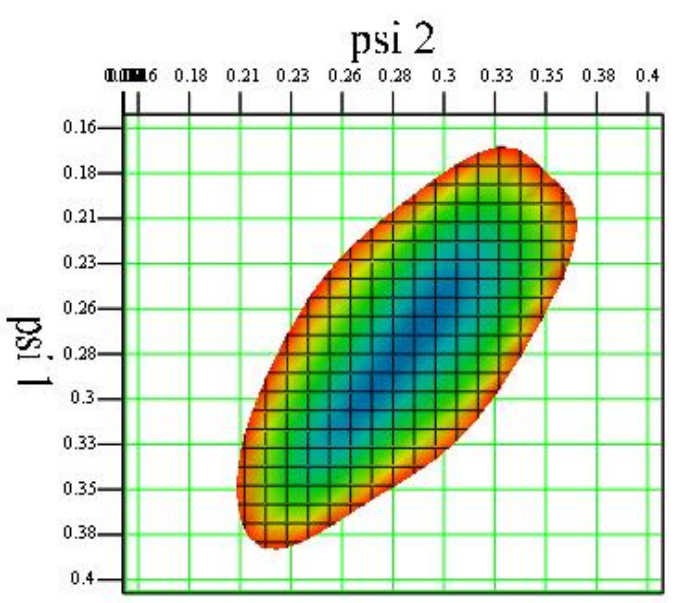

b)

Fig. 9. Dependence of THD of the input current of 24-pulse rectifier on the values of shift angles $\psi_{i}:$ a) 3D chart; b) cross section in the horizontal plane.

To design and operate systems with multi-pulse rectifiers, except THD, the maximum rectifier input current deviation from a sine wave (the vector sum of the highest harmonics in a controlled range of frequencies) is important. Knowledge of the maximum current deviation allows determining of the required characteristics of the active filter that corrects rectifier THD in accordance with the requirements of the standards.

As an example, Fig. 10 shows the diagram of 24-pulse rectifier input current instantaneous values, taking into account the current harmonics up to the 50th. 24-pulse REPS consists of four standardized modules, two of which require matching voltage levels by means of PWM or by means of autotransformers.

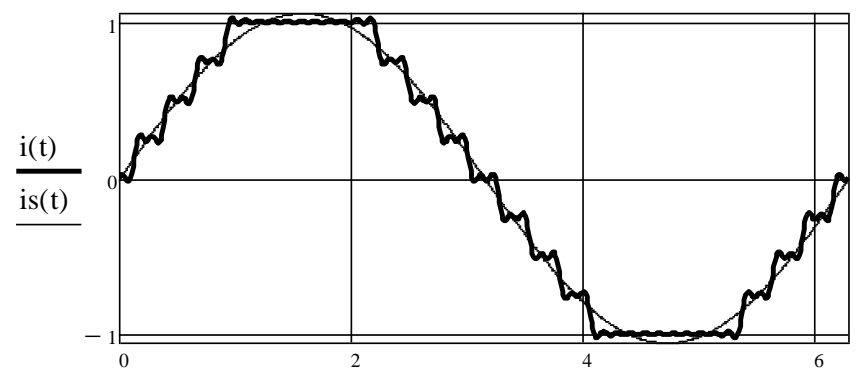

Fig. 10. The instantaneous value of 24-pulse rectifier input current.

Studies of PWM influence on the shape of rectifier input current were performed on the simulation model in Matlab/Simulink environment.

Figure 11 shows the instantaneous value of 24-pulse REPS input current with matching voltage levels by means of PWM. Differences between the input currents of rectifiers with autotransformers and PWM (Fig. 10), (Fig. 11) are negligible.

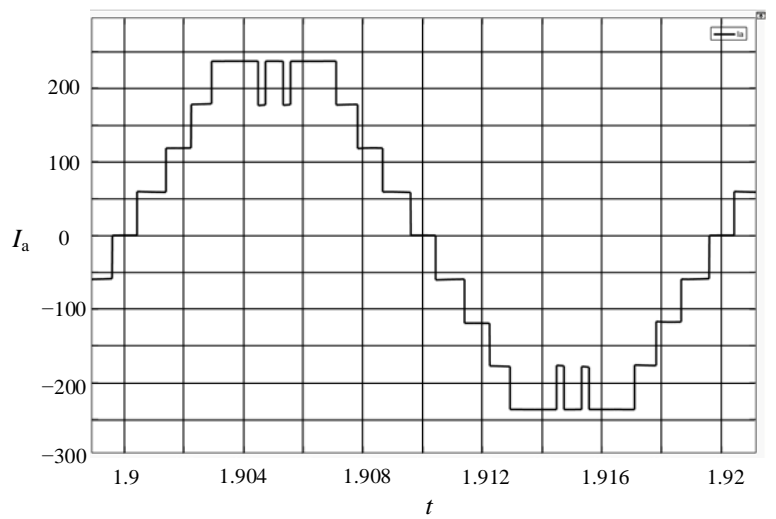

Fig. 11. The instantaneous value of 24-pulse rectifier input current with PWM.

The 24-pulse rectifier input current deviation from the sine wave is shown in Fig. 12. The deviation amplitude does not exceed $16 \%$ of the first current harmonic.

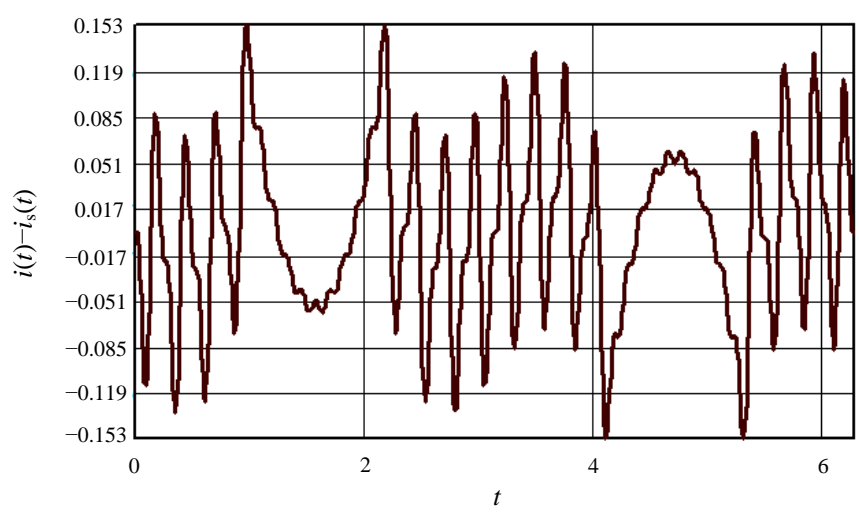

Fig. 12. The 24-pulse rectifier input current deviation from a sine wave. 
Figure 13 shows the dependence of the input current of rectifier maximum deviation from sine wave value on the pulse of rectifier. This relationship is obtained from the analysis of charts similar to charts in Fig. 10.

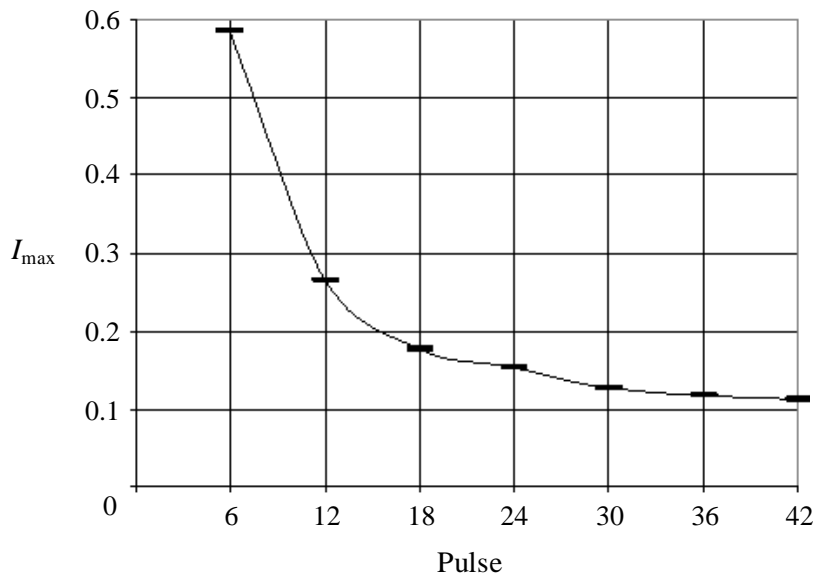

Fig. 13. Dependence of the rectifier input current maximum deviation from sine wave on the rectifier pulse.

The studies show that the input current amplitude value of active or passive ideal corrective filters is $57 \%$ to $12 \%$ of the inverter input current amplitude, depending on rectifier pulses.

\section{INVERTER MODE OF RECTIFIER OPERATION}

When the polarity of output voltage is changed, a rectifier with an electronic phase shift, like a conventional controlled rectifier, can be switched to inverter mode. In this mode, the energy is transferred from source $E_{\mathrm{d}}$ to power supply network. As an example, in Fig. 14 a diagram of the possible use of REPS as a DC/AC converter for PV system energy transferring to an alternating current network is shown.

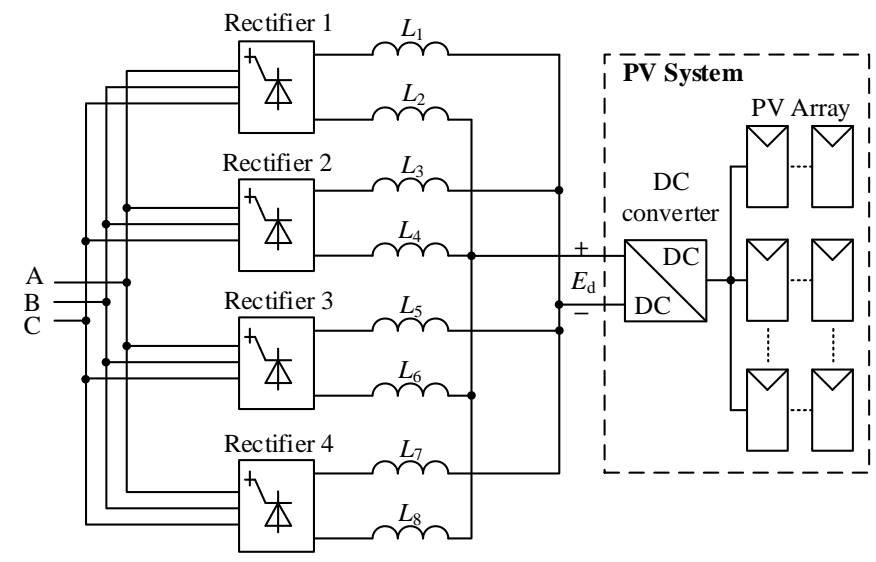

Fig. 14. 24-pulse rectifier with pulse-width modulation in inverter mode.

REPS control has certain features. Usually, the synchronization of a switch control is performed from the lineto-line voltages of corresponding phases. In this case it is impossible to get negative control angles (Fig. 3), (Fig. 5), since the formation of control pulse is necessary at the moment before synchronization takes place (Fig. 3). This problem can be solved by synchronizing the line-to-line voltage of the other phase. For example, to form the angles of control $(-\alpha)$ for "phase $A B$ " switches, you can synchronize with the inverse line- to-line voltage of this phase and form an angle of $(180-\alpha)$. It is possible to reduce the time delay of control system by synchronizing it with voltage $B C$ or the inverse voltage of $C A$ (Fig. 15, a). The minimum angle of control corresponds with the last option and is equal to $(60-\alpha)$ degree.

When switching to inverter mode, the control angle increases by 180 degrees. To reduce the delay time of the system, synchronization can be carried out by inverse voltages with respect to the rectifying mode (Fig. 15, b).

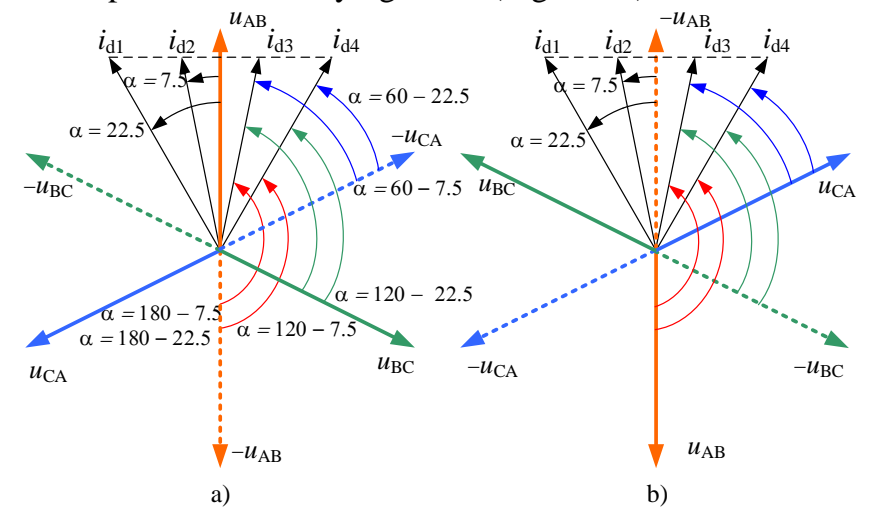

Fig. 15. Vector current diagram of 24-pulse rectifier with electronic phase shift: a) Rectifying mode; b) Inverter mode.

To verify the validity of theoretical statements and conclusions, processes in REPS have been simulated by using Matlab/Simulink environment.

Figure 16 shows the diagrams of input current and voltage of phase $A$ when the 24-pulse converter is operating in rectifier mode.

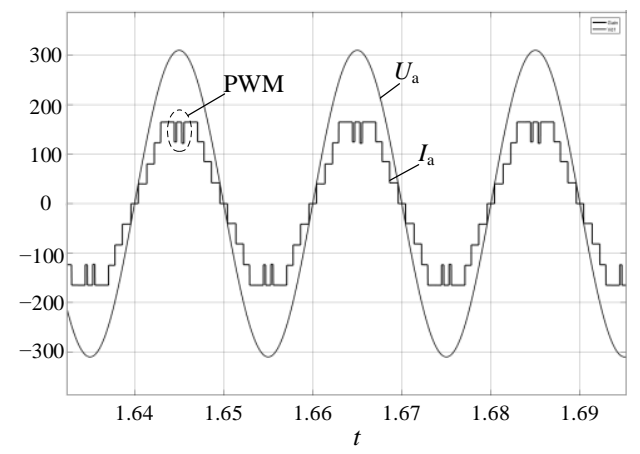

Fig. 16. Diagrams of the input current and voltage of phase A. 24-pulse converter is operating in the rectifier mode.

In Fig. 17, the diagrams of the input current and voltage of phase $A$ are shown when the 24-pulse converter is operating in the inverter mode.

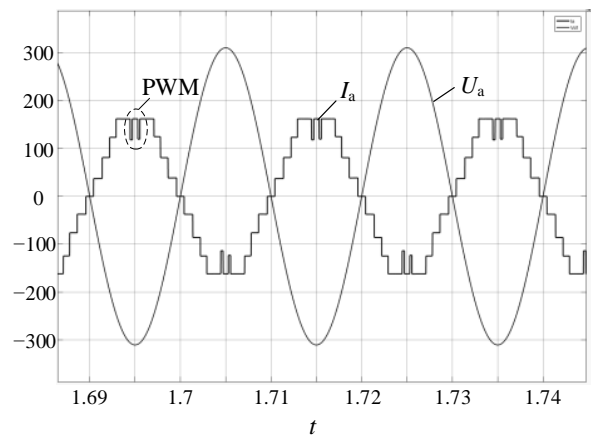

Fig. 17. Diagrams of the input current and voltage of phase A. 24-pulse converter is operating in the inverter mode. 
From Fig. 16 and Fig. 17, we can conclude that the proposed method of REPS control system synchronization is operating. The electronic phase shift allows providing the unity displacement factor and the rated THD of the input current of the converter, both in rectifier and inverter operating modes.

\section{CONCLUSION}

The paper proposes the use of multi-pulse rectifiers with electronic phase shifting and pulse number more than 12 . The base module is a 6-pulse rectifier, with fully controlled switches with reverse blocking capability. Switching frequency is either a match or twice the power line frequency. The proposed solutions allow refusing from electromagnetic phaseshifting devices (power transformers or auto-transformers) and thereby significantly reduce the weight of the device. Unlike PFC systems with high-frequency PWM, the proposed converters significantly differ, as they have better electromagnetic compatibility and the virtual absence of dynamic switching losses of power switches. It is shown, that for the considered class of converters with rectifiers with pulse number from 18 to 36 should be used. For the networks with $I_{\mathrm{SC}} / I_{L}<20$ the use of 24-pulse rectifier with the input filter an installed capacity of less than $1 \%$ of power load is required. A rectifier with electronic phase shifting allows providing the unity displacement factor and the rated THD of input current of the converter, both in rectifier and inverter operating modes.

\section{REFERENCES}

[1] "IEEE Recommended Practice and Requirements for Harmonic Control in Electric Power Systems," in IEEE Std 519-2014 (Revision of IEEE Std 519-1992), pp. 1-29, June $11 \quad 2014$. https://doi.org/10.1109/IEEESTD.2014.6826459

[2] M. Rashid, Power Electronics Handbook, 3nd ed. Boston: Elsevier Inc., 2011, p. 1409.

[3] F. L. Luo and H. Ye, Power Electronics: Advanced Conversion Technologies. Boca Raton: CRC Press, 2010, p. 745.

[4] S. Choi, P. N. Enjeti, and I. J. Pitel, "Polyphase transformer arrangements with reduced $\mathrm{kVA}$ capacities for harmonic current reduction in rectifiertype utility interface," IEEE Transactions on Power Electronics, vol. 11, no. 5, pp. 680-690, Sep. 1996. https://doi.org/10.1109/63.535400

[5] K. Oguchi, G. Maeda, N. Hoshi, and T. Kubata, "Coupling rectifier systems with harmonic cancelling reactors," IEEE Industry Applications Magazine, vol. 7, no. 4, pp. 53-63, 2001. https://doi.org/10.1109/2943.930991

[6] Electromagnetic compatibility $(E M C)-$ Part 3-2: Limits - Limits for harmonic current emissions (equipment input current $<=16 \mathrm{~A}$ per phase), IEC 61000-3-2:2014.

[7] Electromagnetic compatibility (EMC) - Part 3-4: Limits - Limitation of emission of harmonic currents in low-voltage power supply systems for equipment with rated current greater than 16 A, IEC 61000-3-4:1998.

[8] Electromagnetic compatibility (EMC) - Part 3-6: Limits - Assessment of emission limits for the connection of distorting installations to $M V, H V$ and EHV power systems, IEC 61000-3-6:2008.

[9] Electromagnetic compatibility (EMC) - Part 4-19: Testing and measurement techniques - Test for immunity to conducted, differential mode disturbances and signalling in the frequency range $2 \mathrm{kHz}$ to 150 $\mathrm{kHz}$ at a.c. power ports, IEC 61000-4-19:2014.

[10] G. F. Bartak and A. Abart, "EMI of emissions in the frequency range 2 $\mathrm{kHz}-150 \mathrm{kHz}, " 22$ nd International Conference and Exhibition on Electricity Distribution (CIRED 2013), 2013, pp. 1-4. https://doi.org/10.1049/cp.2013.1151

[11] D. A. Paice, Power Electronics Converter Harmonics : Multipulse Methods for Clean Power, Wiley-IEEE Press, 1999, p. 222.
[12] K. Matsui, "A dual thyristor converter reducing harmonics of power supply without input transformer," Electrical Engineering in Japan, vol. 112, no. 3, pp. 143-153, 1992. https://doi.org/10.1002/eej.4391120315

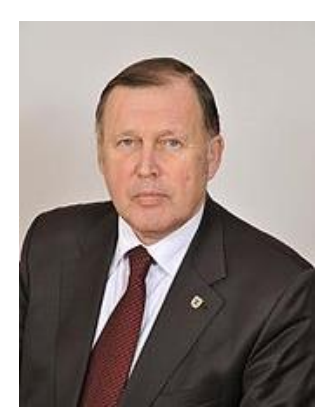

Yevgen I. Sokol was Rector of the National Technical University "Kharkov Polytechnic Institute" in 2015. He has been a corresponding member of the National Academy of Sciences of Ukraine since 2012. He received the Award of S. A. Lebedev National Academy of Sciences of Ukraine in 2001. He is currently a Professor and Head of the Department of Industrial and Biomedical Electronics. He is the author of over 300 scientific works, including 5 monographs, and holds 30 copyright certificates and patents for inventions.

Address: Electric Machine Industry Department, National Technical University "Kharkiv Polytechnic Institute", 2 Kyrpychova Str., Kharkiv 61002, Ukraine.

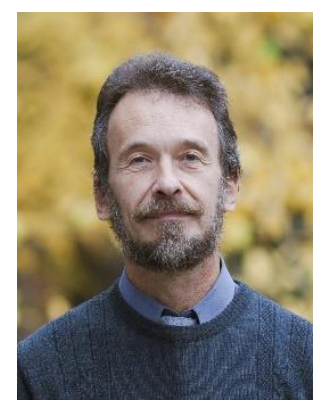

Volodymyr V. Zamaruiev received the Ph.D. degree in electrical engineering from Kharkiv Polytechnic Institute, Ukraine, in 1996. From 1982 to 1995 , he was a research engineer with Industrial Electronics Department of Kharkiv Polytechnic Institute. In 1995, he joined the faculty of National Technical University "Kharkiv Polytechnic Institute" where he currently is a Professor with Industrial and Biomedical Electronics Department. He has published over 150 papers, including 18 patents. $\mathrm{He}$ is a co-author of book "Special-purpose microcontroller system" (Kharkiv, 2007). His interests are power electronics and embedded system design. Much of his current research focuses on dedicated control algorithms and their application to power electronic converter design.

Address: Electric Machine Industry Department, National Technical University “Kharkiv Polytechnic Institute”, 2 Kyrpychova Str., Kharkiv 61002, Ukraine.

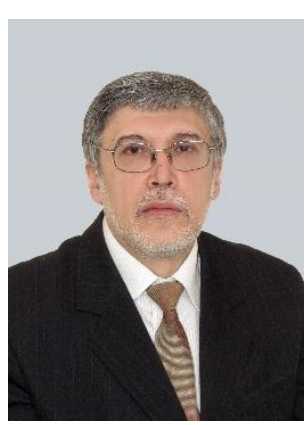

Volodymyr V. Ivakhno received the Ph.D. degree in electrical engineering from Kharkiv Polytechnic Institute, Ukraine, in 1989. From 1982 to 1991 , he was a research engineer in Industrial Electronics Department of Kharkiv Polytechnic Institute. In 1995, he joined the faculty of National Technical University "Kharkiv Polytechnic Institute" where he currently is a Professor with Industrial and Biomedical Electronics Department. $\mathrm{He}$ has published over 140 papers, including 15 patents. His interests include power electronics (power semiconductor switches, soft commutation, optimization of electric power conversion, etc.). Address: Electric Machine Industry Department, National Technical University “Kharkiv Polytechnic Institute”, 2 Kyrpychova Str., Kharkiv 61002, Ukraine.

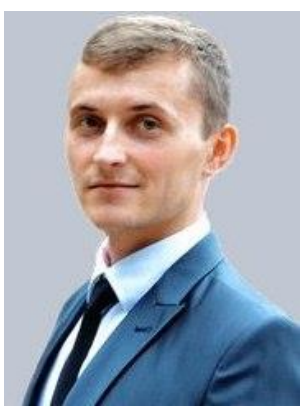

Yurii S. Voitovych received a Master degree in electronic systems from National Technical University "Kharkiv Polytechnic Institute", Ukraine, in 2014. In 2015, he became a postgraduate student with the Department of Industrial and Biomedical Electronics at the Kharkov Polytechnic University. He has published 7 papers, including 1 patent. Address: Electric Machine Industry Department, National Technical University" Kharkiv Polytechnic Institute", 2 Kyrpychova Str., Kharkiv 61002, Ukraine. 